\title{
SNP in the genome-wide association study hotspot on chromosome 9p21 confers susceptibility to diabetic nephropathy in type 1 diabetes
}

\author{
E. Fagerholm • E. Ahlqvist • C. Forsblom • \\ N. Sandholm • A. Syreeni • M. Parkkonen • \\ A. J. McKnight • L. Tarnow • A. P. Maxwell • \\ H.-H. Parving • L. Groop • P.-H. Groop • \\ on behalf of the FinnDiane Study Group
}

Received: 20 December 2011 / Accepted: 20 April 2012 / Published online: 29 May 2012

(C) Springer-Verlag 2012

\begin{abstract}
Aims/hypothesis Parental type 2 diabetes mellitus increases the risk of diabetic nephropathy in offspring with type 1 diabetes mellitus. Several single nucleotide polymorphisms (SNPs) that predispose to type 2 diabetes mellitus have recently been identified. It is, however, not known whether such SNPs also confer susceptibility to diabetic nephropathy in patients with type 1 diabetes mellitus.

Methods We genotyped nine SNPs associated with type 2 diabetes mellitus in genome-wide association studies in the Finnish population, and tested for their association with diabetic nephropathy as well as with severe retinopathy and cardiovascular disease in 2,963 patients with type 1 diabetes mellitus. Replication of significant SNPs was sought in 2,980 patients from three other cohorts.
\end{abstract}

Electronic supplementary material The online version of this article (doi:10.1007/s00125-012-2587-0) contains peer-reviewed but unedited supplementary material, which is available to authorised users.

E. Fagerholm • C. Forsblom • N. Sandholm • A. Syreeni ·

M. Parkkonen - P.-H. Groop $(\bowtie)$

Folkhälsan Institute of Genetics, Folkhälsan Research Center,

Biomedicum Helsinki, University of Helsinki,

Haartmaninkatu 8, POB 63, 00014 Helsinki, Finland

e-mail: per-henrik.groop@helsinki.fi

E. Fagerholm - C. Forsblom • N. Sandholm - A. Syreeni ·

M. Parkkonen $\cdot$ P.-H. Groop

Department of Medicine, Division of Nephrology,

Helsinki University Central Hospital,

Helsinki, Finland

E. Ahlqvist $\cdot$ L. Groop

Department of Clinical Sciences, Diabetes and Endocrinology,

Lund University, Skåne University Hospital,

Malmö, Sweden
Results In the discovery cohort, rs10811661 near gene $C D K N 2 A / B$ was associated with diabetic nephropathy. The association remained after robust Bonferroni correction for the total number of tests performed in this study (OR $1.33[95 \%$ CI $1.14,1.56], p=0.00045, p_{36 \text { tests }}=0.016$ ). In the metaanalysis, the combined result for diabetic nephropathy was significant, with a fixed effects $p$ value of 0.011 (OR 1.15 [95\% CI 1.02, 1.29]). The association was particularly strong when patients with end-stage renal disease were compared with controls (OR 1.35 [95\% CI 1.13, 1.60], $p=0.00038$ ). The same SNP was also associated with severe retinopathy (OR 1.37 [95\% CI 1.10, 1.69] $p=0.0040)$, but the association did not remain after Bonferroni correction $\left(p_{36 \text { tests }}=0.14\right)$. None of the other selected SNPs was associated with nephropathy, severe retinopathy or cardiovascular disease.

A. J. McKnight • A. P. Maxwell

Nephrology Research Group, Queens University of Belfast,

Belfast, UK

L. Tarnow

Steno Diabetes Center,

Gentofte, Denmark

H.-H. Parving

Department of Endocrinology, Rigshospitalet,

Copenhagen, Denmark

P.-H. Groop

Baker IDI Heart and Diabetes Institute,

Melbourne, VIC, Australia 
Conclusions/interpretation A SNP predisposing to type 2 diabetes mellitus, rs10811661 near $C D K N 2 A / B$, is associated with diabetic nephropathy in patients with type 1 diabetes mellitus.

Keywords $C D K N 2 A \cdot$ Diabetic nephropathy · Genetics · rs10811661 . Type 1 diabetes mellitus · Type 2 diabetes mellitus

$\begin{array}{ll}\text { Abbreviations } \\ \text { ACR } & \text { Albumin/creatinine ratio } \\ \text { CDK } & \text { Cyclin-dependent kinase } \\ \text { eGFR } & \text { Estimated GFR } \\ \text { ESRD } & \text { End-stage renal disease } \\ \text { MDRD } & \text { Modification of Diet in Renal Disease } \\ \text { SDR } & \text { Scania Diabetes Registry } \\ \text { SNP } & \text { Single-nucleotide polymorphism } \\ \text { UK-ROI } & \text { All Ireland and Warren 3/UK Genetics } \\ & \text { of Kidneys in Diabetes }\end{array}$

\section{Introduction}

Clustering in families implicates a genetic component of diabetic nephropathy, but so far the specific genes underlying diabetic nephropathy remain largely unknown $[1,2]$. Family studies have furthermore revealed that parental type 2 diabetes mellitus is associated with diabetic nephropathy in offspring with type 1 diabetes mellitus [3, 4]. A positive family history of type 2 diabetes mellitus has also been associated with cardiovascular disease [5] as well as markers of cardiovascular disease [6] in offspring with type 1 diabetes mellitus. Genetic variants or single-nucleotide polymorphisms (SNPs) predisposing to type 2 diabetes mellitus in the Finnish population have recently been identified in large-scale, genome-wide association studies [7, 8]. The question thus arises of whether these SNPs, which predispose to type 2 diabetes mellitus, also predispose to diabetic nephropathy and related complications in patients with type 1 diabetes mellitus. We therefore assessed the impact of a set of SNPs known to influence susceptibility to type 2 diabetes mellitus on diabetic nephropathy as well as diabetic retinopathy and cardiovascular disease in patients with type 1 diabetes mellitus.

\section{Methods}

Discovery cohort All 2,963 patients were part of the ongoing nationwide Finnish Diabetic Nephropathy Study (FinnDiane). Patient recruitment has previously been described elsewhere [9]. In brief, patients took part in the study during a regular visit to their attending physician. Information about concomitant disorders and medication was collected using a standardised questionnaire. Anthropometric measures as well as blood pressure were recorded, and blood and urine samples were collected. The study protocol was approved by the local ethics committees, and the patients gave their written informed consent prior to participation. The study was performed in accordance with the Helsinki Declaration as revised in 2000.

Type 1 diabetes mellitus was defined as an onset of diabetes before the age of 35 years and insulin treatment initiated within 1 year of diagnosis. Renal status was defined based on the AER from at least two out of three timed overnight or $24 \mathrm{~h}$ urine collections. Patients were classified as having normal AER $(<20 \mu \mathrm{g} / \mathrm{min}$ or $<30 \mathrm{mg} / 24 \mathrm{~h})$, microalbuminuria (AER $\geq 20$ and $<200 \mu \mathrm{g} / \mathrm{min}$ or $\geq 30$ and $<300 \mathrm{mg} / 24 \mathrm{~h}$ ), macroalbuminuria (AER $\geq 200 \mu \mathrm{g} / \mathrm{min}$ or $\geq 300 \mathrm{mg} / 24 \mathrm{~h}$ ), or end-stage renal disease (ESRD; undergoing dialysis or having received a kidney transplant). If a patient was on antihypertensive treatment at the time of investigation, a careful review of the AER data was performed in order to ensure that no regression from a higher to a lower stage of albuminuria had occurred due to the intervention. Of the 2,963 patients included in the study, 1,618 had a normal AER (54.6\%), 465 had microalbuminuria (15.7\%), 425 had macroalbuminuria (14.3\%), 433 had ESRD (14.6\%) (see electronic supplementary material [ESM] Table 1), and $22(0.7 \%)$ were unclassified.

We defined patients with microalbuminuria, macroalbuminuria and ESRD $(n=1,323)$ as cases in the analyses for association with diabetic nephropathy. Controls with a normal AER were required to have a duration of diabetes of at least 15 years $(n=1,234)$. The GFR was estimated with the original Modification of Diet in Renal Disease (MDRD) 4 formula until 2002 and after that with the revised MDRD4 formula [10]. MDRD4 data were available for 2,674 of the 2,963 patients included in the study. For analysis of retinopathy, laser treatment was used to define patients $(n=1,167)$ with severe retinopathy. Controls were required to have a duration of diabetes of more than 15 years and no signs of retinopathy $(n=566)$. Cardiovascular disease was defined as a history of myocardial infarction, coronary artery bypass, stroke, amputation or peripheral vascular disease. In the analysis of cardiovascular disease, 298 patients had a history of cardiovascular disease, while 655 patients older than 45 years with no known cardiovascular events served as controls.

Replication cohorts For replication, 892 Danish patients with type 1 diabetes mellitus from the Steno Diabetes Centre were studied. Type 1 diabetes mellitus was defined as an onset of diabetes before 35 years of age and insulin initiation within 1 year of diagnosis. The patients were originally studied at a baseline visit between 1993 and 2001 at the 
Steno Diabetes Center. For the current analysis, we used all available prospective information collected until 1 September 2006. At that stage, 376 patients had a normal AER $(<30 \mathrm{mg} / 24 \mathrm{~h})$ with a duration of diabetes of more than 15 years, 60 patients had microalbuminuria (progression from normoalbuminuria), 365 patients had macroalbuminuria, and 91 patients had ESRD (defined as dialysis or transplantation). As in the FinnDiane cohort, patients with microalbuminuria, macroalbuminuria and ESRD were included as cases $(n=516)$ in the analyses of diabetic nephropathy, and controls had a duration of diabetes of more than 15 years.

Replication was also performed in patients with type 1 diabetes mellitus recruited as part of the All Ireland and Warren 3/UK Genetics of Kidneys in Diabetes (UK-ROI) collections as previously described [11]. Briefly, cases $(n=747)$ were patients diagnosed with overt diabetic nephropathy (proteinuria $>0.5 \mathrm{~g} / 24 \mathrm{~h}$ ), hypertension and retinopathy. Individuals recruited to the control group $(n=812)$ had had type 1 diabetes mellitus diagnosed at less than 31 years of age and had no evidence of renal complications after at least 15 years of diabetes.

A third replication cohort came from the Scania Diabetes Registry (SDR), Sweden [12], which registers all individuals with diabetes in the Malmö region in Southern Sweden. Type 1 diabetes mellitus was defined by age of onset ( $\leq 35$ years), presence of antibodies against GAD and low C-peptide levels. Where information was incomplete, the physician's own classification was used. Patients of non-Scandinavian origin were excluded from the analysis. Stages of nephropathy were defined by AER, as in the FinnDiane cohort, or by albumin/ creatinine ratio (ACR): normal ACR $<2.5 \mathrm{mg} / \mathrm{mmol}$ (men) or $3.5 \mathrm{mg} / \mathrm{mmol}$ (women); microalbuminuria $\geq 2.5 \mathrm{mg} / \mathrm{mmol}$ and $<25 \mathrm{mg} / \mathrm{mmol}$ (men) or $\geq 3.5 \mathrm{mg} / \mathrm{mmol}$ and $<35 \mathrm{mg} / \mathrm{mmol}$ (women); macroalbuminuria $\geq 25 \mathrm{mg} / \mathrm{mmol}$ (men) or $\geq 35 \mathrm{mg} / \mathrm{mmol}$ (women). ESRD was defined as estimated GFR (eGFR) $<15 \mathrm{ml} / \mathrm{min}$, estimated by the MDRD4 formula. The cohort included 90 patients with microalbuminuria, 107 with macroalbuminuria, 34 with ESRD and 298 controls with a normal ACR or AER and more than 15 years' duration of type 1 diabetes mellitus.

Genotyping We selected nine SNPs (ESM Table 2) known to influence susceptibility to type 2 diabetes mellitus in the Finnish population [7, 8]. Genotyping in the discovery cohort was performed with Sequenom iPlex MassARRAY (Sequenom Inc., San Diego, CA, USA) at the University of Eastern Finland (Kuopio, Finland). Genotyping of rs5219 in the KCNJ11 gene was not successful on this platform, and the SNP was excluded from further analyses. The mean call rate was 98.6\%, and all SNPs were in Hardy-Weinberg equilibrium $(p>0.01)$. All replication cohorts were genotyped with a commercially available TaqMan assay (Applied Biosystems, Warrington, UK), and the respective genotypes rates were $99.7 \%$ for UK-ROI, $99.2 \%$ for Danish patients and $98.5 \%$ for SDR.

Statistical analysis We estimated the effect of selected SNPs predisposing to type 2 diabetes by linear or logistic regression analyses corrected for sex, age at onset of diabetes and duration of diabetes using an additive genetic model. All statistical analyses were performed with PLINK version 1.07 (http://pngu.mgh.harvard.edu/ purcell/PLINK) [13]. A two-tailed $p$ value of 0.05 was considered significant. Multiple testing was addressed by Bonferroni correction. Power calculations were carried out using the Genetic Power Calculator (http://pngu.mgh.harvard.edu/ purcell/gpc/) [14]. The study had greater than $80 \%$ power to detect associations between SNPs with risk allele frequencies of $0.15-0.85$, and for diabetic nephropathy $(\alpha=0.05)$ in the FinnDiane cohort, assuming a prevalence of nephropathy of $30 \%$, and a relative risk of 1.3 for heterozygote carriers and 1.7 for homozygote carriers. In the meta-analysis, we used Cochran's $Q$ statistic to detect heterogeneity across studies $(p<0.10)$. The inconsistency index $I^{2}$ was used to quantify heterogeneity. An $I^{2}$ of $25 \%, 50 \%$ and $75 \%$ was regarded as low, moderate and high heterogeneity, respectively [15].

\section{Results}

Clinical characteristics for FinnDiane cases and controls for each analysed trait are shown in ESM Table 1. The nine selected SNPs in or near CDKAL1 (rs7754840 and rs7756992), CDKN2A/B (rs10811661), HHEX (rs1111875), IGF2BP2 (rs4402960 and rs1470579), PPARG (rs1801282), SLC30A8 (rs13266634) and TCF7L2 (rs7903146) were all in Hardy-Weinberg equilibrium $(p>0.01)$, as seen in ESM Table 2. We analysed the association between these nine SNPs, known to influence susceptibility to type 2 diabetes mellitus, and diabetic nephropathy in 2,963 individuals with type 1 diabetes mellitus. Of the SNPs tested, SNP rs10811661 near $C D K N 2 A / B$ was significantly associated with diabetic nephropathy (Table 1). After Bonferroni correction for the total number of tests performed in this study (nine SNPs $\times$ four phenotypes $=36$ ), this $\mathrm{SNP}$, rs10811661 in the $C D K N 2 A / B$ locus, remained significant (OR 1.33, $p=0.016$ ).

We further analysed the association with renal function using eGFR. All patients with ESRD were excluded from these analyses. No new significant associations were detected. However, SNP rs10811661 near $C D K N 2 A / B$ was also significant on a nominal level for this trait $(p=0.0026$; ESM Table 3), showing a decrease in eGFR of on average 2.63 units per risk allele. 
Table 1 Odds ratios and $p$ values of all SNPs for diabetic nephropathy after adjustment for sex, age at onset and duration of type 1 diabetes mellitus in the FinnDiane cohort

\begin{tabular}{|c|c|c|c|c|c|c|c|c|c|}
\hline SNP & Gene & Risk allele & Freq $^{\mathrm{a}}$ (cases) & Freq $^{\mathrm{a}}$ (controls) & $n$ & OR & $p$ & $p_{9}$ tests & $p_{36 \text { tests }}$ \\
\hline rs 7754840 & $C D K A L 1$ & $\mathrm{C}$ & 0.35 & 0.36 & 2,515 & $0.92(0.82,1.04)$ & 0.18 & 1 & 1 \\
\hline rs7756992 & $C D K A L 1$ & G & 0.33 & 0.35 & 2,509 & $0.89(0.79,1.01)$ & 0.071 & 0.64 & 1 \\
\hline rs10811661 & $C D K N 2 A B$ & $\mathrm{~T}$ & 0.87 & 0.83 & 2,518 & $1.33(1.14,1.56)$ & 0.00045 & 0.0041 & 0.016 \\
\hline rs1111875 & HHEX & $\mathrm{C}$ & 0.55 & 0.53 & 2,525 & $1.06(1.14,1.20)$ & 0.26 & 1 & 1 \\
\hline rs1470579 & $I G F 2 B P 2$ & $\mathrm{C}$ & 0.30 & 0.29 & 2,521 & $1.02(0.90,1.15)$ & 0.81 & 1 & 1 \\
\hline rs4402960 & $I G F 2 B P 2$ & $\mathrm{~T}$ & 0.29 & 0.29 & 2,521 & $1.02(0.90,1.15)$ & 0.80 & 1 & 1 \\
\hline rs1801282 & PPARG & $\mathrm{C}$ & 0.82 & 0.82 & 2,515 & $1.02(0.88,1.19)$ & 0.79 & 1 & 1 \\
\hline rs13266634 & SLC $30 A 8$ & $\mathrm{C}$ & 0.61 & 0.62 & 2,519 & $0.98(0.87,1.19)$ & 0.71 & 1 & 1 \\
\hline rs7903146 & $T C F 7 L 2$ & $\mathrm{~T}$ & 0.19 & 0.20 & 2,517 & $0.93(0.80,1.07)$ & 0.32 & 1 & 1 \\
\hline
\end{tabular}

${ }^{a}$ Frequency of the risk allele

In addition, we evaluated the association between cardiovascular disease, retinopathy and the nine selected SNPs. The clinical characteristics of the cases and controls for severe retinopathy as well as cardiovascular disease are shown in ESM Table 1. In these analyses, $C D K N 2 A / B$ rs10811661 was associated with severe retinopathy (OR 1.37 [95\% CI 1.10-1.69], $p=0.0040$ ). However, the association did not remain significant after Bonferroni correction $\left(p_{36 \text { tests }}=0.14\right)$. None of the nine SNPs was associated with cardiovascular disease (ESM Table 4).

To further study the microvascular complications, we created, in a post-hoc analysis, a microvascular phenotype (diabetic nephropathy and/or severe retinopathy). As in the analyses of severe retinopathy, controls were required to have no signs of retinopathy. In this analysis, the CDKN2AB rs10811661 SNP was associated with microvascular complications (ESM Table 5).

We further analysed $C D K N 2 A / B$ rs 10811661 in terms of diabetic nephropathy in the replication cohorts (Table 2). The clinical characteristics of cases and controls in each cohort can be seen in ESM Table 1. In the meta-analysis, the combined result of the FinnDiane patients with the three replication cohorts was significant with a fixed effects $p$ value of 0.011 and an OR of 1.15. However, the SNP was not significantly associated with diabetic nephropathy in the separate replication cohorts. Of note, the OR for diabetic nephropathy in Danish patients was, although not significant, in a direction consistent with the Finnish results. There was, however, moderate heterogeneity between the studies: $p$ value for Cochran's $Q$ statistic $=0.05$ and $I^{2}=61.6 \%$.

Because of the observed heterogeneity between the studies, we decided to evaluate the association between this particular SNP and various degrees of diabetic nephropathy (microalbuminuria, macroalbuminuria and ESRD) in more detail. The association of rs10811661 with microalbuminuria, macroalbuminuria and ESRD was significant in all case-control settings in the FinnDiane cohort. When these results were combined with the results from the other cohorts, the strongest association was observed when ESRD patients were compared with controls (OR 1.35, $p=0.00038$; Fig. 1). Notably, there was a trend of decreasing heterogeneity when more serious degrees of diabetic nephropathy were analysed. For instance, when patients with microalbuminuria were compared with controls, high heterogeneity was observed $\left(I^{2}=83.3 \%, p_{\text {Cochran }}=0.0024\right)$, in contrast to only low heterogeneity when ESRD patients were compared with controls $\left(I^{2}=18.7 \%, p_{\text {Cochran }}=0.30\right)$.

Table 2 Odds ratios and $p$ values for rs 10811661 for diabetic nephropathy adjusted for sex, age at onset and duration of type 1 diabetes in each cohort

\begin{tabular}{|c|c|c|c|c|c|c|}
\hline Patient cohort & Risk allele & $n$ (cases/controls) & Risk allele frequency (cases/controls) & $p_{\mathrm{HWE}}$ & OR $(95 \% \mathrm{CI})$ & $p$ value \\
\hline FinnDiane & $\mathrm{T}$ & $2,518(1,323 / 1,234)$ & $0.85(0.87 / 0.83)$ & 0.03 & $1.33(1.14,1.56)$ & 0.00045 \\
\hline UK-ROI & $\mathrm{T}$ & $1,555(747 / 808)$ & $0.83(0.82 / 0.83)$ & 0.66 & $0.98(0.81,1.19)$ & 0.85 \\
\hline Danish/Steno & $\mathrm{T}$ & $892(516 / 376)$ & $0.84(0.84 / 0.82)$ & 0.14 & $1.18(0.91,1.53)$ & 0.21 \\
\hline Swedish/SDR & $\mathrm{T}$ & $529(231 / 298)$ & $0.82(0.81 / 0.83)$ & 0.18 & $0.91(0.65,1.26)$ & 0.57 \\
\hline Meta-analysis & $\mathrm{T}$ & $5,494(2,817 / 2,716)$ & NA & NA & $1.15(1.02,1.29)^{\mathrm{a}}$ & $0.011^{\mathrm{a}}$ \\
\hline
\end{tabular}

$p$ value and OR for fixed effects. Cochran's $Q$ test, $p=0.05$; heterogeneity index, $I^{2}=61.6 \%$

Controls with a duration of diabetes of less than 15 years were excluded

HWE, Hardy-Weinberg equilibrium; NA, not applicable 


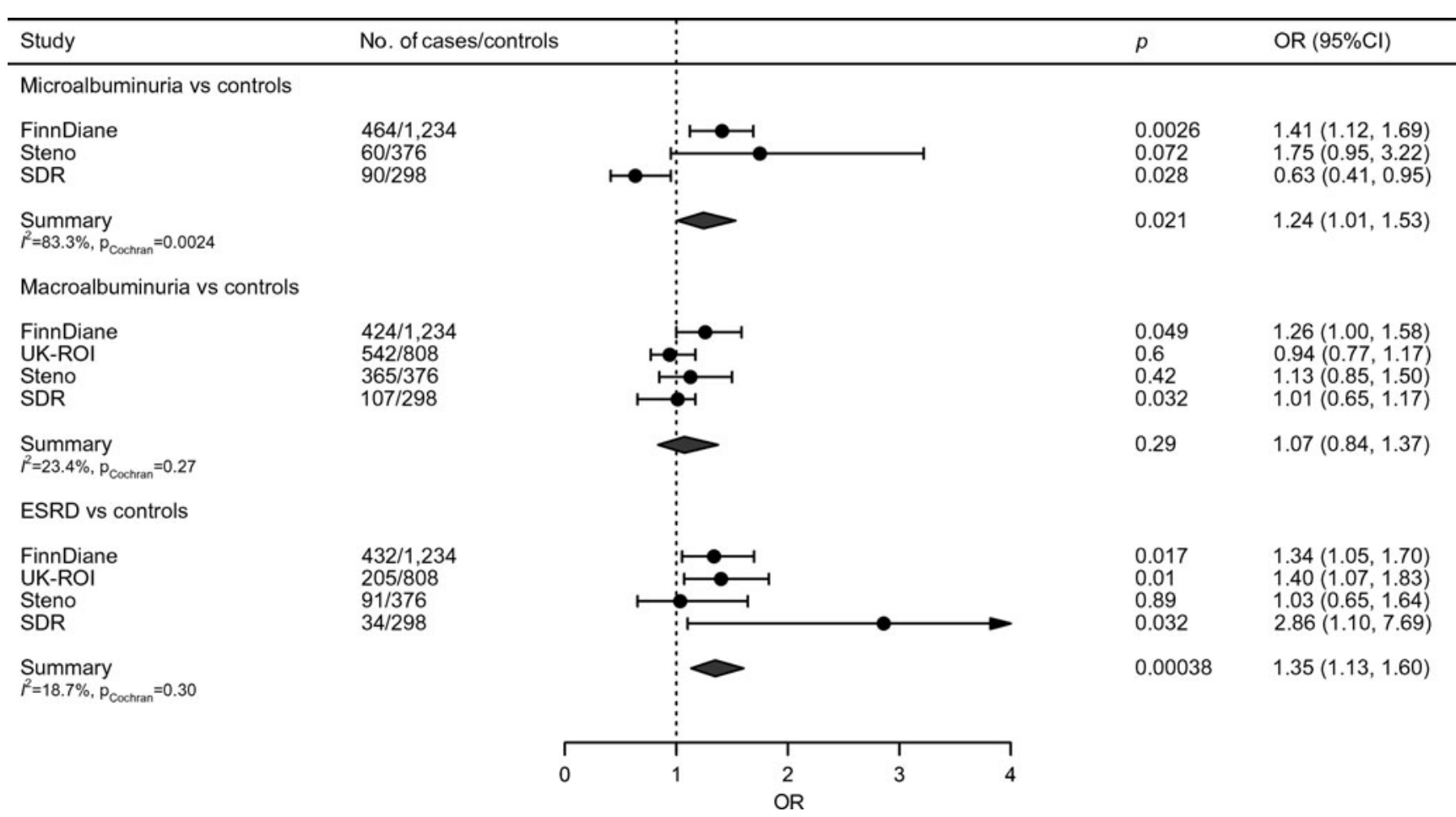

Fig. 1 ORs and $p$ values for rs10811661 and subtypes of diabetic nephropathy in each cohort

\section{Discussion}

In this study, we find that a SNP (rs10811661) near CDKN2A/ $B$, identified from genome-wide association studies on type 2 diabetes mellitus in Finnish patients, is associated with diabetic nephropathy in patients with type 1 diabetes mellitus. The association remained after robust correction for multiple testing in the discovery cohort. In the meta-analysis, the association was significant, although moderate heterogeneity was observed between the studies ( $p$ value for Cochran's $Q$ test $<0.10, I^{2}>$ $50 \%$ ). This SNP was also associated with severe retinopathy but not with cardiovascular disease. Although the association with severe retinopathy did not remain after Bonferroni correction, we believe that the results regarding severe retinopathy in the discovery cohort support our findings concerning nephropathy.

The observed heterogeneity in the meta-analysis may have several explanations. One plausible explanation could be variability in the phenotype. Although the definition of nephropathy was consistent over the cohorts, the distribution of patients with microalbuminuria, macroalbuminuria and ESRD differed. For example, cases of diabetic nephropathy in the FinnDiane cohort consisted of equal numbers of patients with microalbuminuria (35\%), macroalbuminuria (32\%) and ESRD (33\%), whereas a majority of the cases in the other cohorts consisted of patients with macroalbuminuria (Fig. 1). To address this issue, we analysed the different stages of diabetic nephropathy separately. In the meta-analysis, we noted that less heterogeneity (18.7\%) occurred when ESRD patients were compared with controls, suggesting that the ESRD phenotype was the most homogenous one across the cohorts (Fig. 1). Thus, the moderate heterogeneity seems to originate from the other phenotypes, microalbuminuria in particular. The strong association observed in the meta-analysis when ESRD patients were compared with controls (OR 1.35, $p=0.00038$ ) supports our initial findings that this SNP is related to diabetic nephropathy.

As well as trying to dissect the observed heterogeneity, we also tried to minimise type I and type II errors in this study. The occurrence of false-negative results (type II errors) in genetic association studies can lead to difficulties in reproducing the original findings in the replication cohorts. In particular, when sample size is small, falsenegative results are more likely to occur. It has been reported that sample size is critical, especially when studying the effect of common genetic variants with modest effect size on complex disease [16], such as the susceptibility variants identified in genome-wide association studies on type 2 diabetes mellitus. However, with the FinnDiane sample size $(n=$ $2,505)$ we had over $80 \%$ power to detect an association between the included SNPs and diabetic nephropathy. Similarly, with the sample sizes in the Danish and UK-ROI replication cohorts, we had at least $80 \%$ power to detect the same association. The Swedish sample size had only $53 \%$ power to detect the same association. However, in the combined analyses, we had a well-powered sample size of over 5,000 patients. 
As well as false-negative results, the occurrence of false positives (type I errors) in genetic association studies can be problematic and lead to spurious findings that are consequently difficult to reproduce in other cohorts. For example, multiple testing can increase the risk of false-positive findings [16]. To minimise false positives arising from multiple hypotheses testing, we used a robust Bonferroni correction in which we corrected for not only the number of SNPs included, but also the phenotypes tested. There are also other potential causes, such as ethnic admixture, of false positives in genetic association studies. However, all patients in the discovery cohort as well as in the replication cohorts were of northern European descent.

The SNP rs10811661 is located on chromosome 9p21, a hotspot region for genome-wide association studies, that has been associated with a broad range of age-related diseases besides type 2 diabetes, such as coronary artery disease [17], myocardial infarction [18], abdominal aortic aneurysm [19] and various forms of cancer, for example melanoma [20], glioma [21, 22] and breast cancer [23]. Although associated with diseases of similar pathogenesis, these associations have almost without exception been independent of each other. For example, the SNP rs10811661 that predisposes to type 2 diabetes mellitus does not seem to affect susceptibility to cardiovascular disease, whereas SNPs that predispose to cardiovascular disease have no impact on susceptibility to type 2 diabetes [24, 25]. In concordance with earlier studies, we did not observe any association between rs10811661 and cardiovascular disease. Interestingly, a recent study tested SNPs known to predispose to cardiovascular disease in this region for their association with diabetic nephropathy, but observed no such association [26].

Intriguingly, modest evidence for linkage to the $9 \mathrm{p} 21$ region has also been detected in a linkage analysis on nondiabetic ESRD in African-American families [27]. This linkage region includes rs10811661 but also other SNPs that predispose to cardiovascular disease, cancer, etc. Unfortunately, no fine-mapping was performed, and therefore we cannot be certain that the susceptibility to non-diabetic ESRD in African-American families is mediated by this particular SNP.

SNP rs10811661 is located over $100 \mathrm{~kb}$ downstream of the closest genes $C D K N 2 B$ and $C D K N 2 A$. Even closer, approximately $13 \mathrm{~kb}$ away, is a long stretch of non-coding DNA called ANRIL (also known as $C D K N 2 B-A S 1$ ). It was recently reported that $A N R I L$ is involved in epigenetic silencing of $C D K N 2 A$ [28]. $C D K N 2 A$ and $C D K N 2 B$ encode the cyclin-dependent kinase (CDK) inhibitors p16INK4a and p15INK4b, respectively. Both p16INK4a and p15INK4b are important regulators of the cell cycle by negatively regulating CDK4 and CDK6, two promoters of cell proliferation. Particularly, p16INK4a has been extensively studied and shown to be important in cellular senescence and ageing in various organs [29], including the kidney [30]. The production of this protein increases not only with age, but also with degree of glomerular disease [31], and interestingly decreases in the BBDP rat, an autoimmune model of type 1 diabetes, after treatment with ACE inhibitors [32]. Furthermore, kidneys from patients with type 2 diabetic nephropathy show increased levels of p16INK4a [33].

Although a family history of type 2 diabetes has been linked to diabetic nephropathy in offspring with type 1 diabetes, none of the other SNPs predisposing to type 2 diabetes mellitus, apart from rs10811661, was significantly associated with diabetic nephropathy in patients with type 1 diabetes mellitus in this study. The PPARG variant has already been reported to predict ESRD and mortality in patients with type 1 diabetes mellitus [34]. In our crosssectional study, however, the PPARG variant was not associated with diabetic nephropathy.

One key strength of our study was the large cohort of well-characterised individuals with type 1 diabetes that it included. The presence of the phenotype was also robust and well harmonised between the different cohorts, although the distribution of the different stages of nephropathy varied between them. This potential bias was, however, partly addressed by analysing the different stages of nephropathy separately. One could argue that the choice to include microalbuminuria with the cases with diabetic nephropathy added to the heterogeneity observed in the study. It is true that only about $30 \%$ of microalbuminuric patients with type 1 diabetes mellitus develop macroalbuminuria within the following 10 years $[35,36]$, but it is also true that those patients already at the stage of microalbuminuria show signs of diabetic kidney disease in their kidney biopsies [37]. Therefore, we decided to include them as cases in our analyses.

Another potential limitation is that we included only nine of the nearly 40 SNPs that have to date been identified in genome-wide association studies on type 2 diabetes. However, these nine SNPs were identified in the first wave of genome-wide association studies on type 2 diabetes mellitus in 2007, and may thus have a larger effect size than those identified in later large-scale meta-analyses, which were designed to identify SNPs of smaller effect size. Another important reason why we included this set of SNPs was that they were identified in genome-wide association studies with northern European populations and were thus known to work in these populations.

In conclusion, we find that a SNP known to influence susceptibility to type 2 diabetes mellitus, located near the $C D K N 2 A / B$ genes, is associated with diabetic nephropathy in patients of northern European descent with type 1 diabetes. Additional studies are required to understand the role of this locus in the pathogenesis of diabetic nephropathy and 
determine whether this finding is true also in other ethnic cohorts.

Acknowledgements We would like to acknowledge all the physicians and nurses at each participating study centre (see the ESM) and all the patients participating in the study.

Funding The study was supported by grants from the Folkhälsan Research Foundation, the Wilhelm an Else Stockmann Foundation, the Liv and Hälsa Foundation, the Finnish Medical Society (Finska Läkaresällskapet), the European Comission (HEALTH-F2-2008223211 CEED3) and the European Union's Seventh Framework Program (FP7/2007-2013) for the Innovative Medicine Initiative under grant agreement No. IMI/115006 (the SUMMIT consortium). E. Ahlqvist was funded by a grant from the EU consortium ENGAGE. The funders had no role in study design, in the collection, analysis and interpretation of data, in the writing of the report or in the decision to submit the manuscript for publication.

Duality of interest P.-H. Groop has received grants from Eli Lilly and Roche and lecture fees from Boehringer-Ingelheim, Eli Lilly, Genzyme, Novartis, Novo Nordisk and MSD. The present study is not financially supported by a pharmaceutical company. All other authors declare that there is no duality of interest associated with this manuscript.

Contribution statement EF, CF, P-HG and LG conceived and designed the study. All authors participated in the analysis or interpretation of the data. EF, CF, P-HG and EA drafted the manuscript. All authors revised the article critically and approved the final version to be published.

\section{References}

1. Harjutsalo V, Katoh S, Sarti C, Tajima N, Tuomilehto J (2004) Population-based assessment of familial clustering of diabetic nephropathy in type 1 diabetes. Diabetes 53:2449-2454

2. Seaquist ER, Goetz FC, Rich S, Barbosa J (1989) Familial clustering of diabetic kidney disease. Evidence for genetic susceptibility to diabetic nephropathy. N Engl J Med 320:1161-1165

3. Fagerudd JA, Pettersson-Fernholm KJ, Gronhagen-Riska C, Groop PH (1999) The impact of a family history of type II (noninsulin-dependent) diabetes mellitus on the risk of diabetic nephropathy in patients with type I (insulin-dependent) diabetes mellitus. Diabetologia 42:519-526

4. Hadjadj S, Duengler F, Torremocha F et al (2007) Maternal history of type 2 diabetes is associated with diabetic nephropathy in type 1 diabetic patients. Diabetes Metab 33:37-43

5. Erbey JR, Kuller LH, Becker DJ, Orchard TJ (1998) The association between a family history of type 2 diabetes and coronary artery disease in a type 1 diabetes population. Diabetes Care 21:610-614

6. Mäkimattila S, Ylitalo K, Schlenzka A et al (2002) Family histories of type II diabetes and hypertension predict intima-media thickness in patients with type I diabetes. Diabetologia 45:711-718

7. Scott LJ, Mohlke KL, Bonnycastle LL et al (2007) A genome-wide association study of type 2 diabetes in Finns detects multiple susceptibility variants. Science $316: 1341-1345$

8. Diabetes Genetics Initiative of Broad Institute of Harvard and MIT, Lund University, and Novartis Institutes of BioMedical Research,
Saxena R, Voight BF et al (2007) Genome-wide association analysis identifies loci for type 2 diabetes and triglyceride levels. Science 316:1331-1336

9. Thorn LM, Forsblom C, Waden J et al (2009) Effect of parental type 2 diabetes on offspring with type 1 diabetes. Diabetes Care 32:63-68

10. Levey AS, Coresh J, Greene T et al (2006) Using standardized serum creatinine values in the modification of diet in renal disease study equation for estimating glomerular filtration rate. Ann Intern Med 145:247-254

11. McKnight AJ, Patterson CC, Pettigrew KA et al (2010) A GREM1 gene variant associates with diabetic nephropathy. J Am Soc Nephrol 21:773-781

12. Lindholm E, Agardh E, Tuomi T, Groop L, Agardh CD (2001) Classifying diabetes according to the new WHO clinical stages. Eur J Epidemiol 17:983-989

13. Purcell S, Neale B, Todd-Brown K et al (2007) PLINK: a tool set for whole-genome association and population-based linkage analyses. Am J Hum Genet 81:559-575

14. Purcell S, Cherny SS, Sham PC (2003) Genetic Power Calculator: design of linkage and association genetic mapping studies of complex traits. Bioinformatics 19:149-150

15. Higgins JP, Thompson SG, Deeks JJ, Altman DG (2003) Measuring inconsistency in meta-analyses. BMJ 327:557-560

16. Newton-Cheh C, Hirschhorn JN (2005) Genetic association studies of complex traits: design and analysis issues. Mutat Res 573:54-69

17. McPherson R, Pertsemlidis A, Kavaslar N et al (2007) A common allele on chromosome 9 associated with coronary heart disease. Science 316:1488-1491

18. Helgadottir A, Thorleifsson G, Manolescu A et al (2007) A common variant on chromosome 9p21 affects the risk of myocardial infarction. Science 316:1491-1493

19. Gretarsdottir S, Baas AF, Thorleifsson G et al (2010) Genomewide association study identifies a sequence variant within the DAB2IP gene conferring susceptibility to abdominal aortic aneurysm. Nat Genet 42:692-697

20. Bishop DT, Demenais F, Iles MM et al (2009) Genome-wide association study identifies three loci associated with melanoma risk. Nat Genet 41:920-925

21. Wrensch M, Jenkins RB, Chang JS et al (2009) Variants in the CDKN2B and RTEL1 regions are associated with high-grade glioma susceptibility. Nat Genet 41:905-908

22. Shete S, Hosking FJ, Robertson LB et al (2009) Genome-wide association study identifies five susceptibility loci for glioma. Nat Genet 41:899-904

23. Turnbull C, Ahmed S, Morrison J et al (2010) Genome-wide association study identifies five new breast cancer susceptibility loci. Nat Genet 42:504-507

24. Helgadottir A, Thorleifsson G, Magnusson KP et al (2008) The same sequence variant on 9p21 associates with myocardial infarction, abdominal aortic aneurysm and intracranial aneurysm. Nat Genet 40:217-224

25. Broadbent HM, Peden JF, Lorkowski S et al (2008) Susceptibility to coronary artery disease and diabetes is encoded by distinct, tightly linked SNPs in the ANRIL locus on chromosome 9p. Hum Mol Genet 17:806-814

26. McKnight AJ, Maxwell AP, Fogarty DG, Sadlier D, Savage DA, Warren 3/UK GoKinD Study Group (2009) Genetic analysis of coronary artery disease single-nucleotide polymorphisms in diabetic nephropathy. Nephrol Dial Transplant 24: 2473-2476

27. Freedman BI, Langefeld CD, Rich SS et al (2004) A genome scan for ESRD in black families enriched for nondiabetic nephropathy. J Am Soc Nephrol 15:2719-2727

28. Yap KL, Li S, Munoz-Cabello AM et al (2010) Molecular interplay of the noncoding RNA ANRIL and methylated histone H3 
lysine 27 by polycomb $\mathrm{CBX} 7$ in transcriptional silencing of INK4a. Mol Cell 38:662-674

29. Krishnamurthy J, Torrice C, Ramsey MR et al (2004) Ink4a/Arf expression is a biomarker of aging. J Clin Invest 114:1299-1307

30. Melk A, Schmidt BM, Takeuchi O, Sawitzki B, Rayner DC, Halloran PF (2004) Expression of p16INK4a and other cell cycle regulator and senescence associated genes in aging human kidney. Kidney Int 65:510-520

31. Sis B, Tasanarong A, Khoshjou F, Dadras F, Solez K, Halloran PF (2007) Accelerated expression of senescence associated cell cycle inhibitor p16INK4A in kidneys with glomerular disease. Kidney Int 71:218-226

32. Wolf G, Wenzel U, Ziyadeh FN, Stahl RA (1999) Angiotensin converting-enzyme inhibitor treatment reduces glomerular p16INK4 and p27Kip1 expression in diabetic BBdp rats. Diabetologia 42:1425-1432
33. Verzola D, Gandolfo MT, Gaetani G et al (2008) Accelerated senescence in the kidneys of patients with type 2 diabetic nephropathy. Am J Physiol Renal Physiol 295:F1563-F1573

34. Jorsal A, Tarnow L, Lajer M et al (2008) The PPAR gamma 2 Pro12Ala variant predicts ESRD and mortality in patients with type 1 diabetes and diabetic nephropathy. Mol Genet Metab 94:347-351

35. Forsblom CM, Groop PH, Ekstrand A, Groop LC (1992) Predictive value of microalbuminuria in patients with insulin-dependent diabetes of long duration. BMJ 305:1051-1053

36. Caramori M, Fioretto P, Mauer M (2000) The need for early predictors of diabetic nephropathy risk: is albumin excretion rate sufficient? Diabetes 49:1399-1408

37. Bangstad HJ, Østerby R, Dahl-Jørgensen K et al (1993) Early glomerulopathy is present in young, type 1 (insulin-dependent) diabetic patients with microalbuminuria. Diabetologia 36:523-529 